\title{
Metanálisis: Relación entre factores psicosociales en el trabajo y absentismo laboral
}

\author{
Meta-analyses: Relation between psychosocial factors in the work and labour absenteeism
}

\author{
Josep Ma Molina Aragonés \\ Llobregat (Barcelona). España. \\ Recibido: 11-06-10 \\ Aceptado: 31-08-10

\section{Correspondencia:} \\ Josep $M^{a}$ Molina Aragonés \\ Sistema Emergències Mèdiques (SEM) \\ C/ Pablo Iglesias, 101-115 \\ 08990 L’Hospitalet de Llobregat (Barcelona). España. \\ Tfno: 932644400 \\ e-correo: josepmariamolina@gencat.cat
}

Servicio Prevención Sistema Emergències Mèdiques (SEM). L'Hospitalet de

Resumen

Introducción: De acuerdo con el modelo de demada-control, la elevada demanda laboral, el bajo control sobre el mismo y de forma muy especial la combinación de ambos, supondría un importante riesgo para la salud. El equilibrio entre demanda y control depende, según este modelo, de la organización del trabajo y no de las características individuales de cada persona, aunque, por supuesto, la influencia del ambiente psicosocial de trabajo puede ser, y de hecho es, moderada por las características de la respuesta individual.

Objetivos: El objetivo del estudio fue analizar de manera sistemática aquellos estudios que relacionaban los efectos que sobre el absentismo tienen los factores psicosociales en el seno de las organizaciones, utilizando como elemento principal de valoración el modelo de demanda-control de Karasek, y efectuar un metanálisis para valorar la relación entre ambos.

Métodos: Se identificaron las publicaciones a partir de las bases de datos electrónicas Medline (2004 hasta julio de 2009), Embase (2004 hasta marzo de 2009), PsycInfo (2004 hasta julio de 2009) y en la Librería Cochrane (2004 hasta julio de 2009), sin restricciones por motivo de lenguaje.

Las palabras claves utilizadas fueron absentismo ("absenteeism”), absentismo por enfermedad ("sickness absence"), psicosocial ("psychosocial"), laboral ("occupational") y combinaciones de las mismas, que se eligieron inicialmente para su inclusión en el metanálisis. Adicionalmente se revisaron las citas mencionadas en los originales seleccionados para detectar otros estudios potencialmente relevantes. De este modo se consideraron relevantes 51 artículos que parecían cumplir con los factores objeto de este análisis. De estos se excluyeron finalmente $2(3,9 \%)$ por falta de datos para efectuar el análisis, $6(11,7 \%)$ por tratarse de estudios repetidos o con doble publicación, 2 (3,9\%) por tratarse de estudios con diseño transversal y los restantes $35(68,6 \%)$ porque su información no era relevante para ser incluidos.

Control: El gráfico Forest (Fig. 2) muestra el resultado del metanálisis: el riesgo relativo de sufrir un episodio de absentismo es estadísticamente significativo, con un valor de 1,36 (CI: 1,02-1,82) (Tabla 2).

Demanda: El riesgo de sufrir un episodio de absentismo no es valorable, con un valor de 1,01 (IC: 0,91-1,11). (Tabla 3). 
Si bien la demanda, como dimensión propia de estos factores psicosociales, no parece una variable relacionada o que influencie el absentismo laboral, el control si que se encuentra asociado a este, de manera reiterada y consistente.

Med Segur Trab (Internet) 2010; 56 (220): 200-219

Palabras clave: Absentismo, Absentismo por enfermedad, Psicosocial, Laboral.

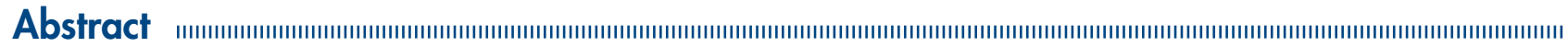

Introduction: In accordance with the model of demand-control, the overhead labour demand, the low control on itself and in a very special way the combination of both, it would suppose an important risk for health. The balance between demand and control depends, just as this model, on the organization of the work and not on the individual characteristics of each person, although, of course, the influence of the working psychosocial environment can be, and in fact is, moderated by the characteristics of the individual answer.

Objectives: The study's objective was to analyse in a systematic way those studies that related the effects over absenteeism that the psychosocial factors have constituted in the enterprises, using as a main element of assessment, the model of demand-control of Karasek, and to make a meta-analyses to evaluated the relation between both of them.

Metbods: There were identified publications from the electronics data bases Medline (2004 to July 2009), Embase (2004 to March 2009), PsycInfo (2004 to July 2009) and in the Bookshop Cochrane (2004 to July 2009), without restrictions motivated by language.

The keyboards used were absenteeism, sickness absence, psychosocial, occupational and combinations of them that were chosen initially by its inclusion on the meta-analyses. Additionally the appointments mentioned were reviewed in the selected originals to detect some other studies potentially relevant.

In this way the ones considered relevant were 51 articles that seemed to fulfil with the object factors of this analyse. Finally there were excluded 2 (3,9\%) because of no dates to effect the analyse, 6 (11,7\%) to treat about repeated studies or with double publication, $2(3,9 \%)$ to treat about repeated studies with transversal design and the rest $35(68,6 \%)$ because its information was not relevant to be included.

Control: The graphic Forest (Fig. 2) shows the meta-analyse result: the relative risk of suffering an episode of absenteeism is statistically significant, with a value of 1,36 (CI: 1,02-1,82) (Table 2).

Demand: The risk of suffering an episode of absenteeism is valueless, with a value of 1,01 (IC: 0,91-1,11). (Table 3).

The demand, as an itself dimensioned of these psycho-socials factors, does not seem a related variable or an influence for the occupational absenteeism, the control is really associated to it, repeatedly and consistently.

Med Segur Trab (Internet) 2010; 56 (220): 200-219

Keywords: Absenteeism, sickness absence, psychosocial, occupational. 


\section{INTRODUCCIÓN}

Una eficaz prevención laboral debe tener en cuenta no solo aquellos factores de riesgo que afectan a poblaciones sanas, sino también aquellas que pueden influir en el pronóstico de trabajadores con distrés psicológico. Una de las teorías acerca del estrés laboral más ampliamente utilizada ha sido el modelo conocido de demanda-control. De acuerdo con este modelo, la elevada demanda laboral, el bajo control sobre el mismo y de forma muy especial la combinación de ambos, supondría un importante riesgo para la salud. Karasek observó que los efectos del trabajo podían ser el resultado de la combinación de las demandas psicológicas laborales y de las características estructurales del trabajo relacionadas con la posibilidad de tomar decisiones y usar las propias capacidades, lo que le llevó a proponer un modelo bidimensional que integrase ambos tipos de conclusiones. El modelo demanda-control fue desarrollado para describir y analizar situaciones laborales en las que los estresores son crónicos, y pone totalmente el acento en las características psicosociales del entorno de trabajo. Ha sido el modelo más influyente en la investigación sobre el entorno psicosocial de trabajo, estrés y enfermedad, así como el que presenta mayor evidencia científica a la hora de explicar efectos en la salud.

La demanda es la exigencia psicológica que el trabajo implica para la persona. Básicamente hace referencia a cuanto se trabaja: cantidad o volumen de trabajo, presión de tiempo, nivel de atención, interrupciones imprevistas. En este sentido no se limita al trabajo intelectual sino a cualquier tipo de trabajo.

El control es la dimensión esencial del modelo, puesto que este es un recurso para moderar las demandas del trabajo. Es decir, el estrés no depende tanto de un exceso de demanda, como de la falta de capacidad de control para resolverla, El control hace referencia al como se trabaja y tiene dos componentes: autonomía y desarrollo de habilidades. La primera es la posibilidad de la persona de influenciar decisiones relacionadas con su trabajo, de controlar sus propias actividades. El segundo hace referencia al grado en que el trabajo permite a la persona desarrollar sus propias capacidades: aprendizaje, creatividad...

El equilibrio entre demanda y control depende, según este modelo, de la organización del trabajo y no de las características individuales de cada persona, aunque, por supuesto, la influencia del ambiente psicosocial de trabajo puede ser, y de hecho es, moderada por las características de la respuesta individual.

En relación al concepto de absentismo, resulta difícil realizar una definición, ya que conlleva una serie de comportamientos sin aparente relación entre ellos. Desde la vertiente psicosocial, los elementos que han presentado mayor importancia para el absentismo han sido la motivación y la satisfacción laboral. El absentismo es un fenómeno de gran relevancia para la sociedad en su conjunto pero más aún para la empresa, que le dedica una especial atención y lo investiga desde cuatro puntos de vista: Empresarial y económico, sociológico, pedagógico y psicológico. Este último se centra en la motivación, la satisfacción y las actitudes hacia el puesto del trabajo. Es por esto que la ausencia en el trabajo no se debe únicamente a enfermedad $u$ otros motivos certificados sino que a veces refleja una actitud individual o grupal hacia un sistema inflexible para intentar escapar de su control.

\section{OBJETIVOS}

El objetivo del estudio fue analizar de manera sistemática aquellos estudios que relacionaban los efectos que sobre el absentismo tienen los factores psicosociales en el seno de las organizaciones, utilizando como elemento principal de valoración el modelo de demanda-control de Karasek, y efectuar un metanálisis para valorar la relación entre ambos. 


\section{CRITERIOS PARA LA VALORACIÓN DE LOS ESTUDIOS}

\section{Tipos de estudios}

Por motivos inherentes a la salud laboral, se tuvieron en cuenta estudios observacionales (diseños de cohortes y estudios de casos y controles).

\section{Tipos de participantes}

Individuos en edad laboral, admitiendo esta en los distintos países en que se desarrollaron los estudios.

\section{Tipos de intervención}

La intervención requerida para la valoración de los riesgos psicosociales era el método de demanda-control de Karasek o las correspondientes adaptaciones a cada uno de los países en que se desarrollo el estudio.

\section{Tipos de medida de resultado}

La medida de resultado considerada fue el absentismo medido globalmente, cuando este se consideró una variable continua. Cuando el absentismo se consideró como una variable categórica, no se incluyeron en el análisis aquellos estudios que lo mesuraban como de muy corta duración (inferior a tres días) o bien el absentismo recurrente, independientemente de la duración del mismo.

\section{MÉTODOS}

\section{Estrategia de búsqueda}

Se identificaron las publicaciones a partir de las bases de datos electrónicas Medline (2004 hasta julio de 2009), Embase (2004 hasta marzo de 2009), PsycInfo (2004 hasta julio de 2009) y en la Librería Cochrane (2004 hasta julio de 2009), sin restricciones por motivo de lenguaje.

Las palabras claves utilizadas fueron absentismo ("absenteeism"), absentismo por enfermedad ("sickness absence"), psicosocial ("psychosocial"), laboral ("occupational") y combinaciones de las mismas, que se eligieron inicialmente para su inclusión en el metanálisis. Adicionalmente se revisaron las citas mencionadas en los originales seleccionados para detectar otros estudios potencialmente relevantes.

\section{Selección de estudios}

Se revisaron inicialmente todas las publicaciones identificadas cuyos títulos sugerían una potencial relevancia con el objeto principal de esta revisión, basada en determinar la relación entre absentismo laboral y los factores de riesgo psicosocial. La selección inicial se basó en el cribado de los títulos y la valoración de los abstracts electrónicos cuando estos se encontraban disponibles y parecían satisfacer los criterios de elegibilidad.

Para su inclusión en el análisis se consideró necesario que el periodo de absentismo determinado fuera igual o superior a 3 días y que el método de valoración de los factores psicosociales utilizado fuera el método de demanda-control de Karasek o una adaptación del mismo. Se descartaron los estudios con diseño transversal.

La selección definitiva de los artículos se hizo a partir de la lectura completa de los mismos y utilizando como criterio a tal efecto la escala de Newcastle-Ottawa. Se incluyeron en el análisis definitivo aquellos estudios con una puntuación igual o superior a 6 puntos en dicha escala (Ver anexos). 


\section{Tratamiento de los datos}

Los datos correspondientes al absentismo se obtuvieron de las cifras ofrecidas en los propios artículos. Cuando esto no era posible, estos periodos se estimaron a partir de las cifras reflejadas en los estudios, una vez ajustados los periodos de seguimiento para las diferentes poblaciones y sus correspondientes tasas de absentismo (en personas-año de seguimiento). A tal efecto se tuvo en cuenta el riesgo relativo o la odds ratio de aquellos modelos que se habían ajustado teniendo en cuenta los factores demográficos y laborales.

Para la comparación de los efectos se consideraron como poblaciones de referencia (no expuestas) aquellas con baja demanda laboral o elevado control sobre sus tareas, que se compararon con la población de referencia de máxima exposición (el cuarto cuartil o el tercer tercil) según el diseño de los diferentes estudios al valorar los riesgos psicosociales.

\section{Análisis estadístico}

Para efectuar el metanálisis se ha utilizado el programa Sïnergy-3, mediante un modelo de efectos aleatorios. La heterogeneidad se ha valorado utilizando el estadístico $\mathrm{Q}$ de DerSimonian y Laird, con un nivel de significación $\mathrm{p}<0,05$. Puesto que el número de estudios incluidos en el metanálisis ha sido limitado, la heterogeneidad también se ha valorado utilizando métodos de representación gráfica.

\section{RESULTADOS}

\section{Características de los estudios}

Utilizando los criterios de búsqueda anteriormente mencionados se identificaron inicialmente 35.480 referencias, que quedaron reducidas a 919 al utilizar la combinación de los mismos. Estas fueron cribadas cuando se acreditaba la existencia de relación entre factores psicosociales y absentismo laboral o bien cuando los primeros eran una variable explicativa de este último. De este modo se consideraron relevantes 51 artículos que parecían cumplir con los factores objeto de este análisis. De estos se excluyeron finalmente $2(3,9 \%)$ por falta de datos para efectuar el análisis, $6(11,7 \%)$ por tratarse de estudios repetidos o con doble publicación, $2(3,9 \%)$ por tratarse de estudios con diseño transversal y los restantes $35(68,6 \%)$ porque su información no era relevante para ser incluidos.

De los restantes 6 artículos que fueron incluidos en el análisis, cuyas características se muestran en la tabla 1; 5 fueron publicados en inglés y 1 en italiano y se desarrollaron en su totalidad en territorio Europeo.

Tabla 1. Características de los estudios que asocian los factores psicosociales con el absentismo laboral

\begin{tabular}{|c|c|c|c|c|c|c|}
\hline Referencia & País (Año) & Población & Medida & PS & DMB & Diseño \\
\hline 10-Town & $\begin{array}{l}\text { Finlandia } \\
(2005)\end{array}$ & $\begin{array}{l}\text { Trabajadores de } 8 \\
\text { municipios finlandeses }\end{array}$ & JCQ (Karasek) & 3 años & $>3$ días & Cohortes \\
\hline GAZEL & $\begin{array}{l}\text { Francia } \\
\text { (2005) }\end{array}$ & $\begin{array}{l}\text { Trabajadores de las } \\
\text { compañías nacionales de } \\
\text { gas y electricidad }\end{array}$ & $\begin{array}{l}\text { JCQ (Karasek \& } \\
\text { Johnson) }\end{array}$ & 7 años & Continuo & Cohortes \\
\hline Whitehall II & $\begin{array}{l}\text { Inglaterra } \\
(1996)\end{array}$ & $\begin{array}{l}\text { Trabajadores civiles no } \\
\text { industriales del área de } \\
\text { Londres }\end{array}$ & JCQ (Karasek) & 2,2 años & $>7$ días & Cohortes \\
\hline Belstress & $\begin{array}{l}\text { Bélgica } \\
\text { (2004) }\end{array}$ & $\begin{array}{l}\text { Trabajadores de la } \\
\text { administración pública de } \\
25 \text { empresas belgas }\end{array}$ & JCQ (Karasek) & 1 año & $>27$ días & Cohortes \\
\hline DWECS & $\begin{array}{l}\text { Dinamarca } \\
(2006)\end{array}$ & $\begin{array}{l}\text { Trabajadores de } \\
\text { empresas danesas }\end{array}$ & JCQ (Modificado) & 1 año & $>6$ días & Cohortes \\
\hline Veneto & Italia (2008) & $\begin{array}{l}\text { Trabajadores de industria } \\
\text { y servicios de la región } \\
\text { italiana del Veneto ( } 30 \\
\text { empresas) }\end{array}$ & JCQ (Karasek) & 1 año & 10 días & Caso-control \\
\hline
\end{tabular}

PS: Periodo de seguimiento.

DMB: Duración mínima de la baja laboral. 


\section{Resultado del metanálisis}

\section{a) Asociación entre control laboral y absentismo}

\section{a. 1 Estudios de cohortes}

El análisis de heterogeneidad mediante el test de Der Simonian y Laird, $(\mathrm{Qh}=241,29$ $\mathrm{p}<0,000)$ determina la heterogeneidad de los estudios para obtener un estimador combinado del efecto. No obstante, es conocido que se trata de una prueba con escasa potencia estadística, por lo que un resultado no significativo suele ser insuficiente para concluir que no existe heterogeneidad, de modo que se ha recurrido al gráfico de l'Abbé (Figura 1), para determinar este aspecto. En este, los puntos correspondientes a cada estudio se distribuyen de modo relativamente disperso a lo largo de la recta que divide el gráfico e indica una cierta heterogeneidad de los estudios, lo que efectivamente, dificultará obtener un estimador resultado del metanálisis.

Figura 1. Gráfico L’Abbe. Control laboral y absentismo

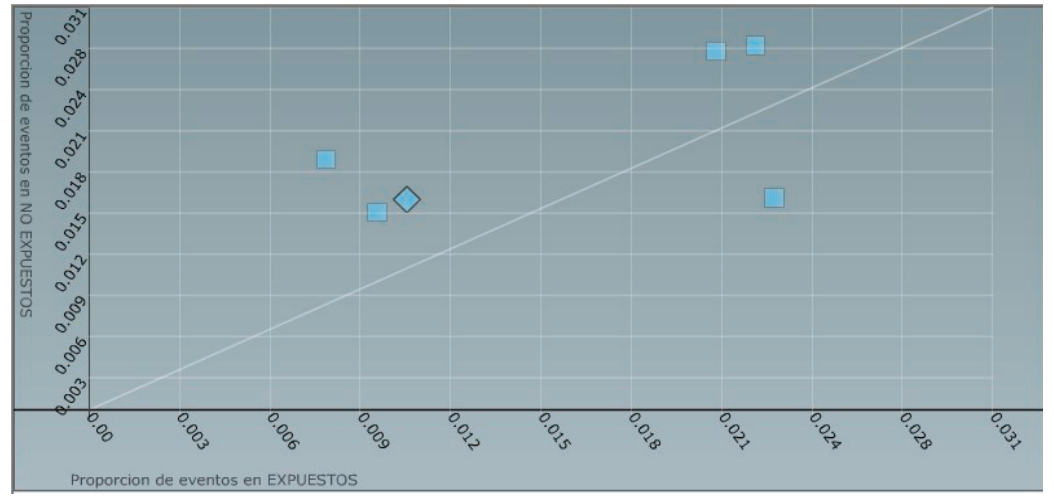

El gráfico Forest (Fig. 2) muestra el resultado del metanálisis: el riesgo relativo de sufrir un episodio de absentismo es estadísticamente significativo, con un valor de 1,36 (CI: 1,02-1,82) (Tabla 2).

Figura 2. Gráfico Forest. Control laboral y absentismo

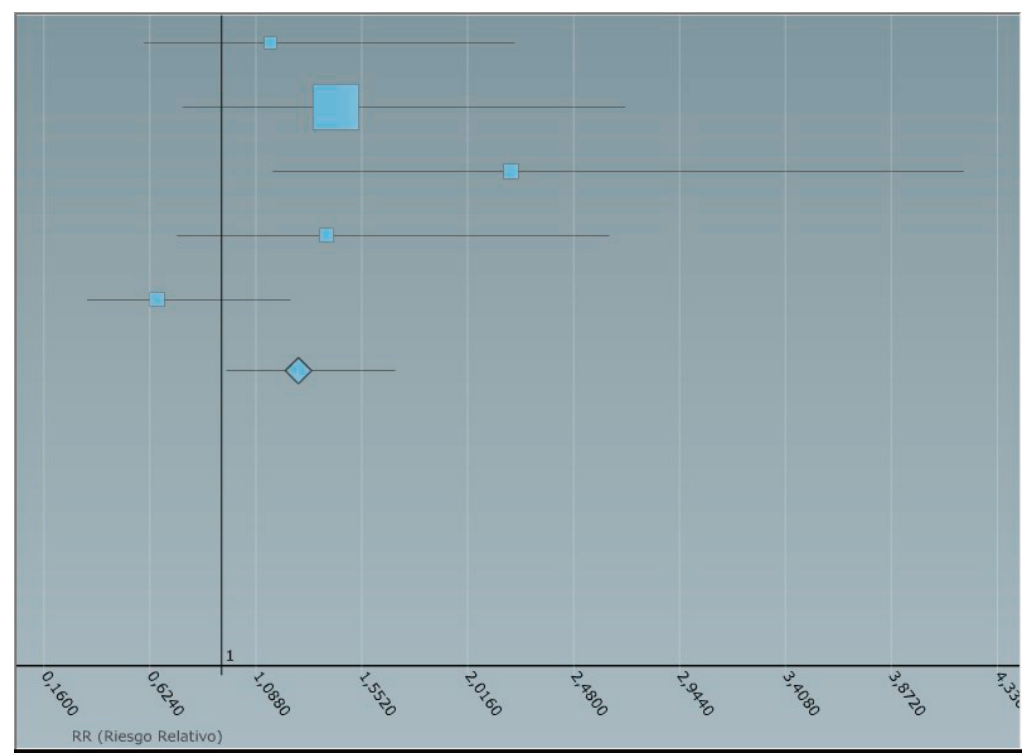


Tabla 2. Resultado del metanálisis control laboral y absentismo

\begin{tabular}{|c|c|c|c|c|c|c|}
\hline $\mathbf{N}^{\circ}$ & Año & Nombre & $\mathbf{R R}$ & IC Inf & IC Sup & EE (LnRR) \\
\hline 1 & 2006 & DWECS & 1,23 & 0,63 & 2,38 & 0,34 \\
\hline 3 & 2005 & GAZEL & 1,54 & 0,82 & 2,91 & 0,32 \\
\hline 4 & 2004 & BELSTRESS & 2,37 & 1,24 & 4,51 & 0,32 \\
\hline 5 & 2002 & 10 TOWN & 1,49 & 0,79 & 2,83 & 0,32 \\
\hline 6 & 1996 & WHITEHALL II & 0,69 & 0,36 & 1,32 & 0,32 \\
\hline \multicolumn{2}{|c|}{ TOTAL } & & 1,36 & 1,02 & 1,82 & 0,14 \\
\hline
\end{tabular}

El gráfico de embudo (Fig. 3) permite analizar la existencia de un sesgo de selección. En este sentido, el aspecto del mismo parece insinuar la posibilidad de la existencia de un sesgo de publicación.

Figura 3. Gráfico Funnel. Control laboral y absentismo

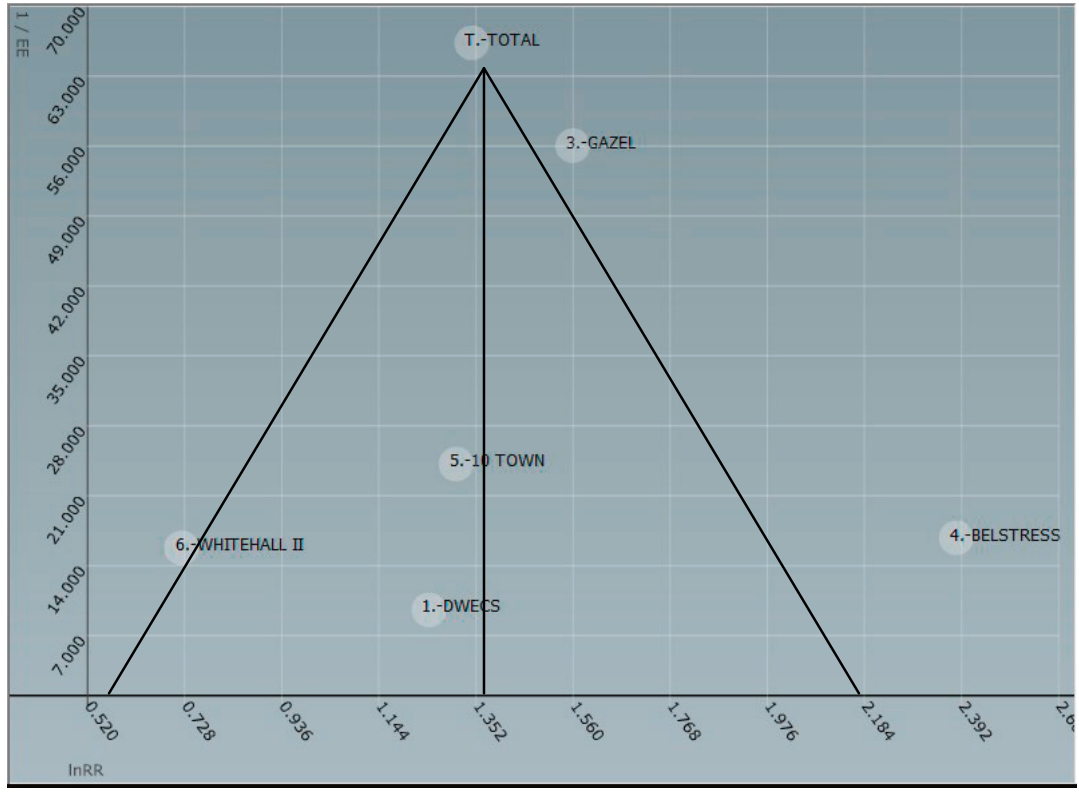

\section{a.2 Estudio de casos-controles}

El estudio de casos controles se trata de un estudio metodológicamente bien diseñado y con buenos criterios de calidad (obtiene una puntuación de 7 en la escala de NewcastleOttawa). Sus resultados van en la misma línea de los obtenidos al efectuar el metanálisis con los estudios de cohortes, por cuanto el control laboral se comporta como un factor de riesgo de sufrir un episodio de absentismo laboral (OR:1,6 [IC: 1,1-2,2])

\section{b) Asociación entre demanda laboral y absentismo}

\section{b. 1 Estudios de cohortes}

Como en el caso previo al analizar el factor de control laboral, el análisis de heterogeneidad me-diante el test de Der Simonian y Laird, $(\mathrm{Qh}=23,43 \mathrm{p}=0,0001)$ determina la heterogeneidad de los estudios, lo que dificulta teóricamente la obtención de un estimador combinado del efecto.

Como se ha comentado con anterioridad, este test es de escasa potencia cuando el número de estudios es bajo, por lo que se recurrirá al gráfico de L'Abbé (Fig. 4) para valorarlo. Según este, los estudios se distribuyen uniformemente alrededor de la línea que divide el gráfico, y puede presumirse la homogeneidad de los estudios incorporados al metanálisis. 
Figura 4. Gráfico L'Abbe. Demanda laboral y absentismo

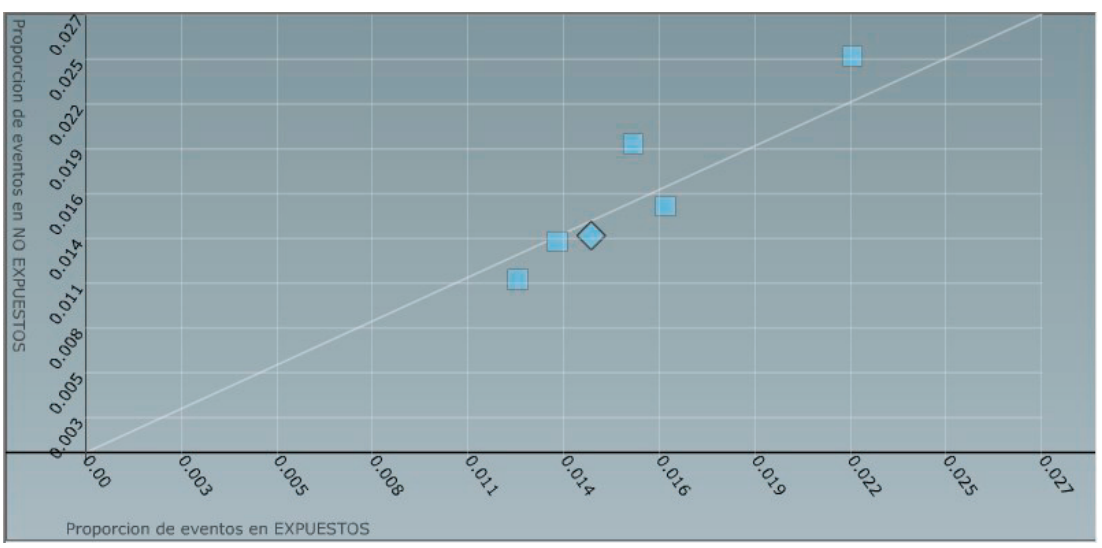

El gráfico Forest (Fig. 5) muestra el resultado del metanálisis. El riesgo de sufrir un episodio de absentismo no es valorable, con un valor de 1,01 (IC: 0,91-1,11). (Tabla 3).

Figura 5. Gráfico Forest. Demanda laboral y absentismo

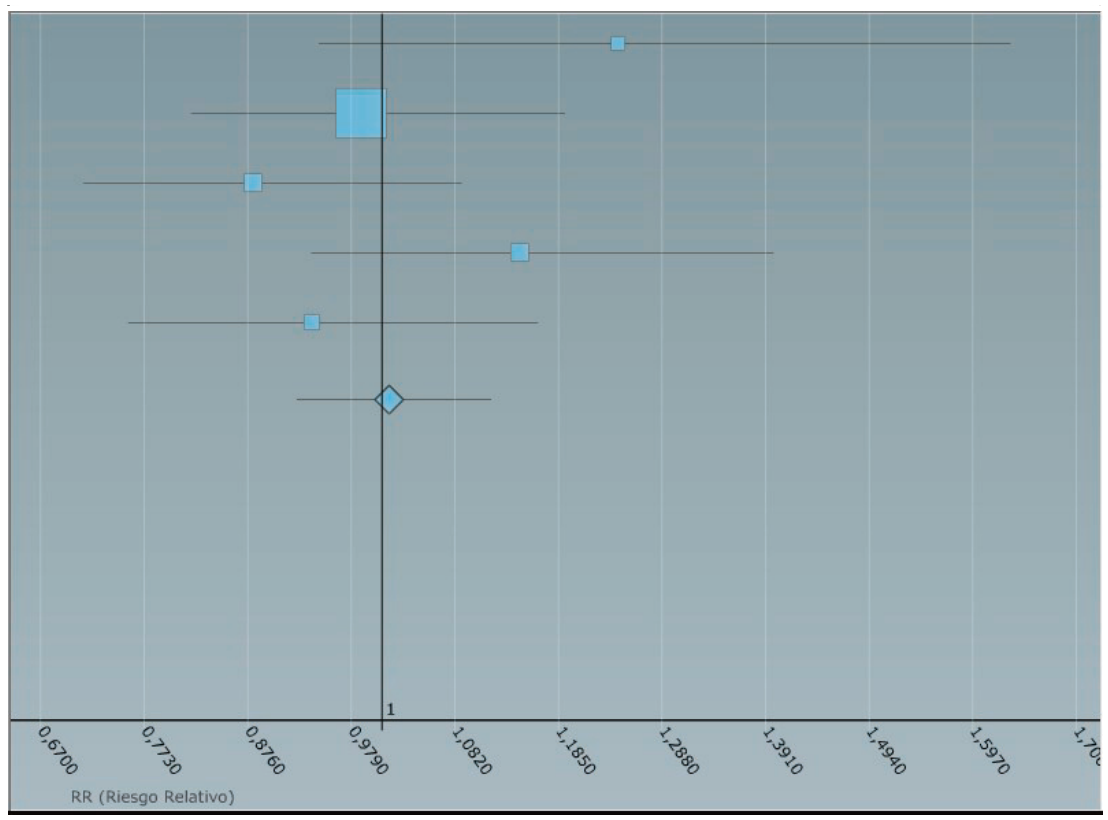

Tabla 3. Resultado del metanálisis demanda laboral y absentismo

\begin{tabular}{|c|c|c|c|c|c|c|}
\hline $\mathbf{N}^{\mathbf{o}}$ & Año & Nombre & $\mathbf{R R}$ & IC Inf & IC Sup & EE (LnRR) \\
\hline 1 & 2006 & DWECS & 1,23 & 0,94 & 1,61 & 0,14 \\
\hline 3 & 2005 & GAZEL & 0,98 & 0,82 & 1,18 & 0,09 \\
\hline 4 & 2004 & BELSTRESS & 0,87 & 0,71 & 1,08 & 0,11 \\
\hline 5 & 2002 & 10 TOWN & 1,13 & 0,93 & 1,38 & 0,10 \\
\hline 6 & 1996 & WHITEHALL II & 0,93 & 0,75 & 1,15 & 0,11 \\
\hline \multicolumn{3}{|c|}{ TOTAL } & $\mathbf{1 , 0 1}$ & 0,92 & $\mathbf{1 , 1 1}$ & 0,05 \\
\hline
\end{tabular}


El gráfico de embudo (Fig. 6) permite analizar la existencia de un sesgo de selección. En este sentido, el aspecto del mismo parece insinuar la posibilidad de la existencia de un sesgo de publicación.

Figura 6. Gráfico Funnel. Demanda laboral y absentismo

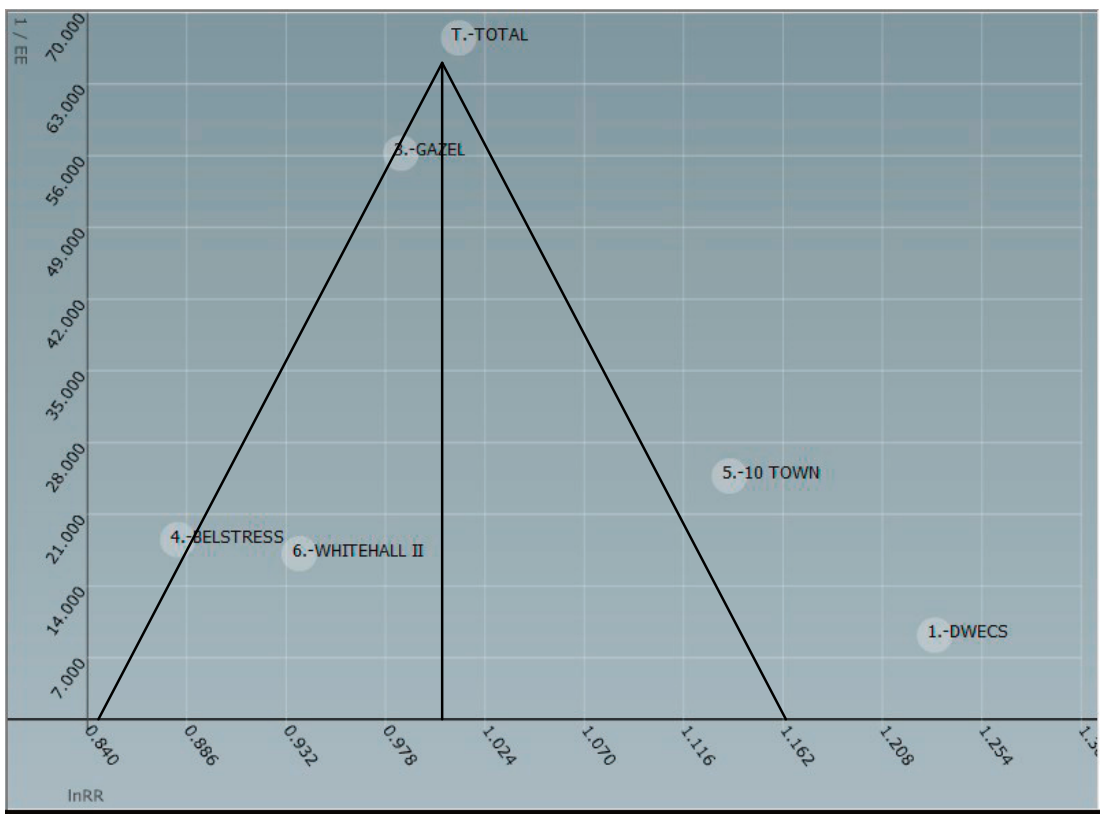

\section{b.2 Estudio de casos-controles}

Respecto a la demanda laboral, el estudio de casos y controles muestra una asociación significativa con el riesgo de sufrir un episodio de absentismo laboral (OR: 1,5 [IC: 1,0-2,2])

\section{DISCUSIÓN}

\section{Hallazgos generales. Interpretación. \\ a) Control laboral y absentismo}

El resultado del metanálisis indica una relación entre el control laboral y el absentismo, con un aumento significativo del riesgo relativo, 1,36 (CI: 1,00-1,78), que también se ve reforzado por el resultado del estudio de casos y controles. Como se ha mencionado anteriormente, el control es la dimensión esencial del modelo, puesto que es el recurso que permite moderar las demandas del trabajo. Este resultado es congruente con el resultado de múltiples estudios 1-4 que relacionan esta dimensión de los factores psicosociales con aumentos significativos del absentismo en los diferentes sectores de producción. La capacidad de decisión del trabajador se ha estimado como el factor de mayor impacto al determinar el absentismo laboral, con una fracción etiológica cercana al $12 \% 5$.

El resultado del metanálisis, aún con las limitaciones que se mencionan en el apartado correspondiente, refuerza la hipótesis de que el control laboral es la principal y más consistente dimensión psicosocial que interviene en el ámbito laboral y que se encuentra íntimamente relacionada con el absentismo, siendo una variable que por si misma puede explicar este complejo fenómeno.

\section{b) Demanda laboral y absentismo}

Los resultados respecto a la asociación ente demanda y absentismo por enfermedad se han mostrado reiteradamente inconsistentes. Así, algunos estudios han mostrado asociación entre baja demanda laboral y aumento del absentismo6,7 . 
El resultado del metanálisis viene a corroborar estos resultados inconsistentes respecto a la relación entre demanda laboral y absentismo, y muestran la inexistencia de relación entre ambos factores: RR:1,01 (IC: 0,91-1,11). El estudio de casos-controles, por su menor potencia, no modifica los resultados de este metanálisis que por su contundente resultado parece clarificar la inconsistencia de estudios previos. En este sentido, si merece la pena rescatar un argumento que relaciona estos resultados con los ciclos económicos, y que puede ser interesante en nuestra actual situación del mercado laboral: cuando existe dificultad para cambiar de puesto de trabajo (es decir, para evitar la demanda de manera radical), es más fácil que emerjan problemas de salud relacionados con esta, que no se darán en circunstancias de mayor flexibilidad del mercado laboral8.

\section{LIMITACIONES}

El estudio puede haber introducido un sesgo de publicación, como parece indican los gráficos de funnel-plot. Este no incluye en el análisis los resultados del estudio de la Cohorte IPAW, que está desarrollando su trabajo en el campo de los riesgos psicosociales: los resultados son consecuentes con los obtenidos en el metanálisis, lo que reforzaría teóricamente el resultado del mismo. No han podido obtenerse los datos correspondientes al absentismo ni efectuar una estimación de los mismos, motivo por el cual no han podido ser incluidos en el análisis. Sin duda se trata de un estudio que, por su magnitud y su diseño, deben ser tenido en cuenta en futuras investigaciones en este ámbito de los riesgos psicosociales.

Otra posible limitación es que el análisis no se ha efectuado teniendo en cuenta motivos de género. En este se ha efectuado una estimación del absentismo global a partir del absentismo de hombres y mujeres, y una distinta distribución de género puede haber introducido una infraestimación del efecto y un sesgo que es preciso tener en cuenta.

Los estudios que relacionan un mayor absentismo femenino con los factores psicosociales son múltiples1,3,4,6,8,9 y muy posiblemente este deba ser un factor a tener en cuenta en este tipo de estudios. También es posible que el mercado laboral suponga un sesgo que deba considerarse en el diseño de estudios de este tipo puesto que las mujeres acceden a puestos de trabajo con menor autonomía y esto pueda incidir de manera significativa tanto en sus tasas de absentismo como en la diferente incidencia de riesgos psicosociales.

\section{RECOMENDACIONES Y CONCLUSIONES}

Los factores psicosociales son cada vez más elementos que deben ser tenidos en cuenta en la gestión preventiva de cualquier organización. Si bien la demanda, como dimensión propia de estos factores psicosociales, no parece una variable relacionada o que influencie el absentismo laboral, el control si que se encuentra asociado a este, de manera reiterada y consistente.

A pesar de las limitaciones del estudio, este demuestra y corrobora la aseveración previa, en el sentido de que el control sobre el trabajo está íntimamente relacionado con el absentismo de media-larga duración, con las connotaciones y los costes que de esto se deriva.

Si las organizaciones quieren mejorar uno de los aspectos más conflictivos de su actual gestión, deben evaluar los riesgos psicosociales, de manera muy especial aquellos aspectos relacionados con el control sobre el trabajo de sus empleados y dotar a estos de estrategias para incidir y modularlo. Este guarda una relación inversamente proporcional con el absentismo y cualquier esfuerzo por aumentarlo supondrá mejoras en los ámbitos económicos y sociales de la organización. 


\section{REFERENCIAS}

1. Kivimäki M, Vahtera J, Pentii J. Et al. Factors underliyng the effect of organisational downsizing on health of employees: longitudinal cohort study. BMJ 2000;320:971-975.

2. Niedhammer I. Chastang JF. David S. Importance of psychosocial work factors on general health outcomes in te national French SUMER survey. Occup Med. 2008. 58: 15-24.

3. Andrea H, Beurskens AJ, Metsemakers JF, van Amelsvoort LG, van den Brandt PA, van Schayck CP. Health problems and psychosocial work environment as predictors of long term sickness absence in employees who visited the occupational physician and/or general practitioner in relation to work: a prospective study. Occup Environ Med. 2003 Apr;60(4):295-300.

4. Munch-Hansen T. Wieclaw J. Agerbo E. Westergard-Nielsen N. Bonde JP. Global measure of satisfaction with psychosocial work conditions versus measures of specific aspects of psychosocial work conditions in explaining sickness absence. BMC Public Health. 2008;8:270

5. Nielsen ML, Rugulies R, Smith-Hansen L, Christensen KB, Kristensen TS. Psychosocial work environment and registered absence from work: estimating the etiologic fraction. Am J Ind Med. 2006 Mar;49(3):187-96.

6. North FM. Syme SL. Feeney A. Shipley M. Marmot M. Psyhosocial work environment and sickness absence among british civil servants: The Whitehall II study. Am J Public Health. 1996; 86:332-340

7. Smulders PGW. Nijhuis FJN. The job demand - job control and absence behavior: results of a 3- year longitudinal study. Work stress. 1999; 13(2): 115-131.

8. Virtanen M, Vahtera J, Pentti J, Honkonen T, Elovainio M, Kivimäki M. Job Strain and psychologic distress. Influence on sickness absence among finnish employees. Am J Prev Med 2007;33 (3):182-187.

9. Labriola M, Lund T, Burr H. Prospective study of pysical and psycosocial risk factors for sickness absence. Occup Med. 2006; 56: 469-474.

10. World Health Organization. The World Health Report 2001. Mental health: new understanding, new hope. Geneva: NMH Communications; 2001.

11. Libro Verde. Mejorar la salud mental de la población. Hacia una estrategia de la Unión Europea en materia de salud mental. Bruselas, 14.10.2005 COM(2005) 484 final.

12. MTAS. INSHT.NTP 603. Riesgo psicosocial: El modelo demanda-control-apoyo social (I).

13. MOOSE Group. Meta-Analysis of Observational studies in Epidemiology. A proposal for reporting. JAMA. $2000 ; 283$ (15); 2008-2012.

14. Iniciativa STROBE. Declaración de la iniciativa STROBE (Strengthening the reporting of observational sudies in Epidemiology): Directrices para la comunicación de estudios observacionales. Gac Sanit. 2008; 22(2); 144-50.

15. Mc Elvenny DM, Armstrong BG, Järup L, Higgins JP. Meta-analysis in occupational epidemiology: a review of practice. Occupational Medicine. 2004; 54: 336-344.

\section{AGRADECIMIENTOS}

A Anne Marie Roche, de Embase por permitir el acceso gratuito a esta base de datos durante tres meses y facilitar de este modo la revisión sistemática. 


\section{ANEXO I. METANÁLISIS. ARTíCULOS EVALUADOS.}

Ala-Mursula L. Vahtera J. Kivimaki M. Kevin MV. Pentti J. Employee control over working times: associations between subjective health and sickness absences. J Epidemiol Community Health. 2002;56:272-2781.

Andrea H, Beurskens AJ, Metsemakers JF, van Amelsvoort LG, van den Brandt PA, van Schayck CP. Health problems and psychosocial work environment as predictors of long term sickness absence in employees who visited the occupational physician and/or general practitioner in relation to work: a prospective study. Occup Environ Med. 2003 Apr;60(4):295-300.

Ariëns GA, Bongers PM, Hoogendoorn WE, van der Wal G, van Mechelen W. High physical and psychosocial load at work and sickness absence due to neck pain. Scand J Work Environ Health. 2002 Aug;28(4):222-31.

Bartis S. Burton K. Main C. A prospective study of psychosocial risk factors and absence due to musculoskeletal disorders-implications for occupational screening. Occupational Medicine.2005;55:375-379.

Bültmann U, Huibers MJ, van Amelsvoort LP, Kant I, Kasl SV, Swaen GM. Psychological distress, fatigue and long-term sickness absence: prospective results from the Maastricht Cohort Study. J Occup Environ Med. 2005 Sep;47(9):941-7.

Clumeck N, Kempenaers C, Godin I, Dramaix M, Kornitzer M, Linkowski P, Kittel F. Working conditions predict incidence of long-term spells of sickness absence due to depression: results from the belstress I prospective study. J Epidemiol Community Health. 2009;63(4):286-92

Christensen KB, Nielsen ML, Rugulies R, Smith-Hansen L, Kristensen TS. Workplace levels of psychosocial factors as prospective predictors of registered sickness absence. J Occup Environ Med. 2005 Sep;47(9):933-40.

Christensen KB. Lund T. Labriola M. Villadsen E. Bültmann U. The fraction of long-term sickness absence attributable to work enviromental factors: prospective results from the Danish Environment Cohort Study. Occup Environ Med. 2007 ; 64 :487-489.

De Croon EM, Blonk RW, Sluiter JK, Frings-Dresen MH. Occupation-specific screening for future sickness absence: criterion validity of the trucker strain monitor (TSM). Int Arch Occup Environ Health. 2005 Feb;78(1):27-34.

Dekkers-Sánchez PM, Hoving JL, Sluiter JK, Frings-Dresen MH. Factors associated with long-term sick leave in sick-listed employees: a systematic review. Occup Environ Med. 2008 Mar;65(3):153-7.

Duijts SFA, Kant IJ, Swaen GMH. Advantages and disadvantages of an objective selection process for early intervention in employees at risk for sickness absence. BMC Public Health. 2007;7:67-74

Duijts SFA, Kant IJ, Swaen GMH, van den Brandt PA, Zeegers MP. A meta-analysis of observational studies identifies predictors of sickness absence. J Clin Epidemiol. 2007 Nov;60(11):1105-15.

Duijts SFA. Kant IJ. Landeweerd JA. Swaen GMH. Predicition of sickness absence: development of a screening instrument. Occup Environ Med. 2006 ;63:564-569

Duijts SFA. Kant IJ. van den Brandt PA. Swaen GMH. Psychometrics and validation of a screening instument for sickness absence. Occupational Medicine. 2008; 58: 413-418

Evans O, Steptoe A. The contribution of gender-role orientation, work factors and home stressors to psychological well-being and sickness absence in male- and female-dominated occupational groups. Soc Sci Med. 2002 Feb;54(4):481-92.

Gimeno D, Benavides FG, Amick BC 3rd, Benach J, Martínez JM. Psychosocial factors and work related sickness absence among permanent and non-permanent employees. J Epidemiol Community Health. 2004 Oct;58(10):870-6.

Head J, Kivimäki M, Martikainen P, Vahtera J, Ferrie JE, Marmot MG. Influence of change in psychosocial work characteristics on sickness absence: The Whitehall II Study. J Epidemiol Community Health. 2006 Jan;60(1):55-61

Ishizaki M, Kawakami N, Honda R, Nakagawa H, Morikawa Y, Yamada Y; Japan Work Stress and Health Cohort Study Group. Psychosocial work characteristics and sickness absence in Japanese employees. Int Arch Occup Environ Health. 2006 Sep;79(8):640-6. ***⿰冫⿰亅⿱丿丶丶

Janssen N. Kant IJ. Swaen GMH. Janssen PPM. Schröer. Fatigue as a predictor of sickness absence: results from the Maastrich cohort study on fatigue at work. Occup Environ Med. 2003 ; 60 (Suppl 1) :71-76.

de Jonge J, Reuvers MM, Houtman IL, Bongers PM, Kompier MA. Linear and nonlinear relations between psychosocial job characteristics, subjective outcomes, and sickness absence: baseline results from SMASH. Study on Musculoskeletal Disorders, Absenteeism, Stress, and Health. J Occup Health Psychol. 2000 Apr;5(2):256-68.

Kivimäki M, Vahtera J, Thomson L, Griffiths A, Cox T, Pentti J. Psychosocial factors predicting employee sickness absence during economic decline. J Appl Psychol. 1997 Dec;82(6):858-72. 
Kivimäki M, Sutinen R, Elovainio M, Vahtera J, Räsänen K, Töyry S, Ferrie JE, Firth-Cozens J. Sickness absence in hospital physicians: 2 year follow up study on determinants. Occup Environ Med. 2001 Jun;58(6):361-6.

Kondo K, Kobayashi Y, Hirokawa K, Tsutsumi A, Kobayashi F, Haratani T, Araki S, Kawakami N. Job strain and sick leave among Japanese employees: a longitudinal study. Int Arch Occup Environ Health. 2006 Mar;79(3):213-9.

Koopmans P. Roelen C. Groothoff J. Risk of future sickness absence in frequent and long-term absentees. Occupational Medicine. 2008;58:268-274

Leka S. Kortum E. A European framework to adress psychosocial hazards. J Occup Health. 2008; 50: $294-296$

Labriola M. Lund T. Burr H. Prospective study of physical and psychosocial risk factor for sickness absence. Occupational Medicine. 2006; 56:469-474.

Lund T, Labriola M, Christensen KB, Bültmann U, Villadsen E, Burr H. Psychosocial work environment exposures as risk factors for long-term sickness absence among Danish employees: results from DWECS/ DREAM. J Occup Environ Med. 2005 Nov;47(11):1141-7. ****

Lund T, Labriola M, Christensen KB, Bültmann U, Villadsen E. Physical work enviroment risk factors for long term sicknes absence: prospective findings among a cohort of 5357 employees in Denmark. BMJ, 2006;332:449-452.

Mastrangelo G, Mattioli S, Baldasseroni A, Bontadi D, Capodicasa E, Marzia V, Mazzi M, Patané P, Torri P, Marangi G, Fadda E, Priolo G, Scoizzato L, Maier E, Campo G, Marchiori L. Occupational injuries and sickness absence: association with job demand, decision latitude, and life style in 2174 workers in the Veneto Region. Med Lav. 2008;99 Suppl 1:31-41.

Melamed S, Ben-Avi I, Luz J, Green MS. Objective and subjective work monotony: effects on job satisfaction, psychological distress, and absenteeism in blue-collar workers. J Appl Psychol. 1995 Feb;80(1):29-42.

Melchior M. Krieger N. Kawachi I. Berkman LF. Niedhammer I. Goldberg M. Work factors and occupationals class disparities in sickness absence: findings from he GAZEL cohort study. American Journal of Public Health. 2005;95(7):1206-12122.

Moreau M, Valente F, Mak R, Pelfrene E, de Smet P, De Backer G, Kornitzer M. Occupational stress and incidence of sick leave in the Belgian workforce: the Belstress study. J Epidemiol Community Health. 2004 Jun;58(6):507-16

Mortelmanns AK. Donceel P. Lahaye D. Bulterys S. Work-related sickness absence and mandatory occupational health surveillance. Occupational Medicine. 2008;58;464-467.

Munch-Hansen T. Wieclaw J. Agerbo E. Westergard-Nielsen N. Bonde JP. Global measure of satisfaction with psychosocial work conditions versus measures of specific aspects of psychosocial work conditions in explaining sickness absence. BMC Public Health. 2008;8:270

Munch-Hansen T. Wieclaw J. Agerbo E. Westergard-Nielsen N. Rosenkilde M. Bonde JP. Sickness absence and workplace levels of satisfaction with psychosocial work conditions at public service workplaces. Am $\mathrm{J}$ Ind Med. 2009;52:153-161.

Niedhammer I, Chastang JF, David S. Importance of psychosocial work factors on general health outcomes in the national French SUMER survey. Occup Med (Lond). 2008 Jan;58(1):15-24.

Nielsen ML, Rugulies R, Smith-Hansen L, Christensen KB, Kristensen TS. Psychosocial work environment and registered absence from work: estimating the etiologic fraction. Am J Ind Med. 2006 Mar;49(3):187-96.

Nieuwenhuijsen K. de Boer AGEM. Verbeek JHAM. Blonk RWB. van Dijk FJH. The Depression Anxiety Stress Scale (DASS): detecting anxiety disorder and depression in employees absent from work because of mental health problems. Occup Environ Med 2003; 60 (Suppl 1):177-182

North FM. Syme SL. Feeney A. Shipley M. Marmot M. Psyhosocial work environment and sickness absence among british civil servants: The Whitehall II study. Am J Public Health. 1996; 86:332-340

Otsuka Y, Takahashi M, Nakata A, Haratani T, Kaida K, Fukasawa K, Hanada T, Ito A. Sickness absence in relation to psychosocial work factors among daytime workers in an electric equipment manufacturing company. Ind Health. 2007 Apr;45(2):224-31.

Peter R, Siegrist J. Chronic work stress, sickness absence, and hypertension in middle managers: general or specific sociological explanations? Soc Sci Med. 1997 Oct;45(7):1111-20.

Roelen C. van der Pol T. Koopmans PC. Groothoff J. Identifying workers at risk of sickness absence by questionnaire. Occupational Medicine 2006;56:442-446

Roelen C. Weites SH. Koopmans PC. van der Klink JJL. Groothoff JW. Sickness absence and psycosocial work conditions: a multilevel study. Occupational Medicine. 2008; 58:425-430.

Sandmark H. Work and family:associations with long-term sick-listings in Swedish women - a case-control study. BMC Public Health. 2007;7:287 
Vasse RM, Nijhuis FJ, Kok G. Associations between work stress, alcohol consumption and sickness absence. Addiction. 1998 Feb;93(2):231-41.

Verhaeghe R, Vlerick P, Gemmel P, Van Maele G, De Backer G. Impact of recurrent changes in the work environment on nurses' psychological well-being and sickness absence. J Adv Nurs. 2006 Dec;56(6):646-56.

Virtanen M, Vahtera J, Pentti J, Honkonen T, Elovainio M, Kivimäki M. Job strain and psychologic distress influence on sickness absence among Finnish employees. Am J Prev Med. 2007 Sep;33(3):182-7.

Virtanen M. Kivimaki M. Elovainio M. Vahtera J. Cooper CL. Contingent employment, health and sickness absence. Scand J Work Environ Health 2001; 27(6):365-372

Virtanen P, Oksanen T, Kivimäki M, Virtanen M, Pentti J, Vahtera J. Work stress and health in primary health care physicians and hospital physicians. Occup Environ Med. 2008;65(5):364-6.

Voss M. Floderus B. Diderichsen F. Physical, psychosocial and organisational factors relative to sickness absence: a study based on sweden post. Occup Environ Med. 2001;58 :178-184

Whitaker SC. The management of sickness absence. Occup Environ Med 2001; 58 :420-424 


\section{ANEXO II. FICHA DE LOS ESTUDIOS INCLUIDOS EN EL METANÁLISIS Y VALORACIÓN SEGÚN ESCALA DE NEWCASTLE - OTTAWA}

\begin{tabular}{|c|c|c|c|c|}
\hline Autores & \multicolumn{4}{|c|}{ Melchior M, Krieger N, Kawachi I, Berkman LF, Niedhammer I, Goldberg M. } \\
\hline Título & \multicolumn{4}{|c|}{$\begin{array}{l}\text { Work factors and occupational class disparities in sickness absence: Findings from the } \\
\text { GAZEL cohort study }\end{array}$} \\
\hline Referencia & \multicolumn{4}{|c|}{ Am J Public Health. 2005;95:1206-12 } \\
\hline Diseño & Cohortes & $\mathbf{N}$ & 11733 & \\
\hline Método & \multicolumn{4}{|c|}{ Basado en el cuestionario de Karasek / Johnson } \\
\hline \multicolumn{5}{|l|}{ Resumen } \\
\hline \multicolumn{5}{|c|}{$\begin{array}{l}\text { Seguimiento ( } 6 \text { años) de los trabajadores de la compañía de gas y electricidad francesa mediante encuesta } \\
\text { anual por correo (tasa de respuesta habitual } 75 \%) \text {, que incluye esfuerzo físico y condiciones psicosociales en } \\
\text { el trabajo. Datos de absentismo obtenidos de los registros propios de la compañía. Se estudiaron todas las } \\
\text { ausencias. En un segundo análisis se discriminaron ausencias cortas ( }<7 \text { días) intermedias ( } 8 \text {-21 días) y largas } \\
(<21 \text { días). Las ausencias se valoraron como tasa por } 100 \text { personas-año. Un 19-21\% del total de ausencias } \\
\text { estaban relacionadas con condiciones laborales adversas. } \\
\text { Limitaciones: Población "mas sana" que el resto de la población que infraestima el absentismo. (Efecto } \\
\text { trabajador sano). Las medidas de exposición fueron tomadas cinco años antes de medir el absentismo y no } \\
\text { se controlaron posibles cambios. }\end{array}$} \\
\hline OR - RR & \multicolumn{4}{|c|}{$\begin{array}{l}\text { o }(\mathrm{N}=8307) \text { Baja decisión: } 1,64(1,54-1,75) \text { Alta demanda: } 0,90(0,83-0,96) \\
\text { + }(\mathrm{N}=2886) \text { Baja decisión: 1,34 (1,23-1,45) }\end{array}$} \\
\hline \multicolumn{5}{|c|}{ ESCALA DE NEWCASTLE-OTTAWA (Estudios Cohortes) } \\
\hline \multicolumn{5}{|c|}{\begin{tabular}{l|l} 
Selección & \\
\end{tabular}} \\
\hline \multirow{4}{*}{\multicolumn{2}{|c|}{$\begin{array}{l}\text { Representatividad de la cohorte } \\
\text { de expuestos }\end{array}$}} & \multicolumn{2}{|c|}{$\begin{array}{l}\text { Verdadera representatividad de los factores psicosociales en la } \\
\text { muestra* }\end{array}$} & \\
\hline & & \multicolumn{2}{|c|}{$\begin{array}{l}\text { Cierta representatividad de los factores psicosociales en la } \\
\text { muestra* }\end{array}$} & $\checkmark$ \\
\hline & & \multicolumn{2}{|c|}{ Grupos seleccionados de trabajadores (pacientes) } & \\
\hline & & \multicolumn{2}{|l|}{ No hay descripción } & \\
\hline \multirow{3}{*}{\multicolumn{2}{|c|}{$\begin{array}{l}\text { Selección de la cohorte de no } \\
\text { expuestos }\end{array}$}} & \multicolumn{2}{|l|}{ Elegida de la misma comunidad que la cohc } & $\checkmark$ \\
\hline & & \multicolumn{2}{|c|}{ Elegida de un modo diferente } & \\
\hline & & \multicolumn{2}{|c|}{$\begin{array}{l}\text { No hay descripción de la selección de la cohorte de no } \\
\text { expuestos }\end{array}$} & \\
\hline \multirow{4}{*}{\multicolumn{2}{|c|}{ Conocimiento de la exposición }} & \multicolumn{2}{|c|}{ Fuente segura (p.e. registro quirúrgico)* } & \\
\hline & & \multicolumn{2}{|l|}{ Entrevista estructurada* } & \\
\hline & & \multicolumn{2}{|l|}{ Cuestionario autoadministrado } & $\checkmark$ \\
\hline & & \multicolumn{2}{|l|}{ No hay descripción } & \\
\hline \multirow{2}{*}{\multicolumn{2}{|c|}{$\begin{array}{l}\text { Demostración de que el evento } \\
\text { de interés no estaba presente al } \\
\text { inicio del estudio }\end{array}$}} & \multicolumn{2}{|l|}{$\mathrm{Si} *$} & $\checkmark$ \\
\hline & & \multicolumn{2}{|l|}{ No } & \\
\hline \multicolumn{5}{|c|}{\begin{tabular}{l|l} 
Comparabilidad & \\
\end{tabular}} \\
\hline & & Análisis de las cohortes por factores sociode & ficos* & \\
\hline $\begin{array}{l}\text { Comparabilic } \\
\text { en la base de }\end{array}$ & $\begin{array}{l}\text { de las cohortes } \\
\text { seño o el análisis }\end{array}$ & $\begin{array}{l}\text { Estudio de controles por otro factor adicion } \\
\text { laborales y educacionales) }\end{array}$ & ctores & $\checkmark$ \\
\hline Resultado & & & & \\
\hline & & Ciego independiente* & & \\
\hline Conocimient & la exposición & Acoplamiento de registro* (Informatización & baja) & $\checkmark$ \\
\hline & & Auto informe & & \\
\hline & & Sin descripción & & \\
\hline $\begin{array}{l}\text { Fue el seguir } \\
\text { suficienteme }\end{array}$ & $\begin{array}{l}\text { to } \\
\text { largo para que }\end{array}$ & $\begin{array}{l}\text { Si (seleccionar un periodo de seguimiento a } \\
\text { resultado esperado }>=6 \text { meses)* (Siete años) }\end{array}$ & o para el & $\checkmark$ \\
\hline sucediera el & lltado & No $(<=6$ meses $)$ & & \\
\hline & & Seguimiento completo - todos los individuo & & \\
\hline Idoneidad de & guimiento de las & $\begin{array}{l}\text { Pérdida de sujetos insuficiente para introduc } \\
\text { Pequeño número de pérdidas }(75 \%) *\end{array}$ & esgo. & $\checkmark$ ? \\
\hline & & Tasa de pérdidas $>\%$, sin descripción de es & didas & $\sqrt{ } ?$ \\
\hline & & Sin referencias & & \\
\hline Total & & & & 6 \\
\hline
\end{tabular}




\begin{tabular}{|c|c|c|c|c|}
\hline Autores & \multicolumn{4}{|c|}{ M Moreau, F Valente, R Mak, E Pelfrene, P de Smet, G De Backer, M Kornitzer } \\
\hline Título & \multicolumn{4}{|c|}{$\begin{array}{l}\text { Occupational stress and incidence of sick leave in the Belgian workforce: the Belstress } \\
\text { study }\end{array}$} \\
\hline Referencia & \multicolumn{4}{|c|}{ J Epidemiol Community Health 2004;58;507-516 } \\
\hline Diseño & Cohortes & $\mathbf{N}$ & \multicolumn{2}{|l|}{20463} \\
\hline Método & \multicolumn{4}{|c|}{ Demanda - control de Karasek } \\
\hline \multicolumn{5}{|l|}{ Resumen } \\
\hline \multicolumn{5}{|c|}{$\begin{array}{l}\text { Seguimiento durante cuatro años en } 25 \text { compañias importantes de Bélgica. La percepción de una elevada } \\
\text { carga laboral (job strain) junto a la falta de soporte social es claramente predictivo de futuro absentismo } \\
\text { laboral. Se excluyeron los seguimientos inferiores a un año y las ausencias por maternidad. }\end{array}$} \\
\hline OR - RR & \multicolumn{4}{|c|}{$\begin{array}{lll}\text { Demanda: } & 0.80(0.63-1.01)[\delta] & 1.18(0.76-1.84)[\text { [ }] \\
\text { Control: } & 3.29(2.47-4.34)\left[{ }^{\lambda}\right] & 1.73(1.16-2.58)[+] \\
\end{array}$} \\
\hline \multicolumn{5}{|c|}{ ESCALA DE NEWCASTLE-OTTAWA (Estudios Cohortes) } \\
\hline \multicolumn{5}{|l|}{ Selección } \\
\hline \multirow{4}{*}{\multicolumn{2}{|c|}{$\begin{array}{l}\text { Representatividad de la cohorte } \\
\text { de expuestos }\end{array}$}} & \multicolumn{2}{|c|}{$\begin{array}{l}\text { Verdadera representatividad de los factores psicosociales en la } \\
\text { muestra* }\end{array}$} & \\
\hline & & \multicolumn{2}{|c|}{$\begin{array}{l}\text { Cierta representatividad de los factores psicosociales en la } \\
\text { muestra * }\end{array}$} & $\checkmark$ \\
\hline & & \multicolumn{2}{|c|}{ Grupos seleccionados de trabajadores (pacientes) } & \\
\hline & & \multicolumn{2}{|c|}{ No hay descripción } & \\
\hline \multirow{3}{*}{\multicolumn{2}{|c|}{$\begin{array}{l}\text { Selección de la cohorte de no } \\
\text { expuestos }\end{array}$}} & \multicolumn{2}{|c|}{ Elegida de la misma comunidad que la cohorte de expuestos* } & $\checkmark$ \\
\hline & & \multicolumn{2}{|l|}{ Elegida de un modo diferente } & \\
\hline & & \multicolumn{2}{|c|}{$\begin{array}{l}\text { No hay descripción de la selección de la cohorte de no } \\
\text { expuestos }\end{array}$} & \\
\hline \multirow{4}{*}{\multicolumn{2}{|c|}{ Conocimiento de la exposición }} & \multicolumn{2}{|c|}{ Fuente segura (p.e. registro quirúrgico)* } & \\
\hline & & \multicolumn{2}{|l|}{ Entrevista estructurada* } & \\
\hline & & \multicolumn{2}{|l|}{ Cuestionario autoadministrado } & $\checkmark$ \\
\hline & & \multicolumn{2}{|l|}{ No hay descripción } & \\
\hline \multirow{2}{*}{\multicolumn{2}{|c|}{$\begin{array}{l}\text { Demostración de que el evento de } \\
\text { interés no estaba presente al inicio } \\
\text { del estudio }\end{array}$}} & \multicolumn{2}{|l|}{$\mathrm{Si} *$} & $\checkmark$ \\
\hline & & \multicolumn{2}{|l|}{ No } & \\
\hline Comparabili & & & & \\
\hline & & Análisis de las cohortes por factores sociode & ficos* & $\checkmark$ \\
\hline la base del di & ño o el análisis & $\begin{array}{l}\text { Estudio de controles por otro factor adicion } \\
\text { laborales y educacionales) }\end{array}$ & tores & $\checkmark$ \\
\hline Resultado & & & & \\
\hline & & Ciego independiente* & & \\
\hline Conocimients & e la exposición & Acoplamiento de registro* (Informatización & aja) & $\checkmark$ \\
\hline & & Auto informe & & \\
\hline & & Sin descripción & & \\
\hline $\begin{array}{l}\text { Fue el seguin } \\
\text { suficientemer }\end{array}$ & $\begin{array}{l}\text { nto } \\
\text { largo para que }\end{array}$ & $\begin{array}{l}\mathrm{Si} \text { (seleccionar un periodo de seguimiento a } \\
\text { resultado esperado }>=6 \text { meses)* (Un año) }\end{array}$ & o para el & $\checkmark$ \\
\hline sucediera el 1 & & No $(<=6$ meses $)$ & & \\
\hline & & Seguimiento completo - todos los individuo & & \\
\hline Idoneidad de & eguimiento de las & $\begin{array}{l}\text { Pérdida de sujetos insuficiente para introdu } \\
\text { Pequeño número de pérdidas }(95 \%) *\end{array}$ & esgo. & $\checkmark$ \\
\hline & & Tasa de pérdidas $>\%$, sin descripción de es & didas & \\
\hline & & Sin referencias & & \\
\hline Total & & & & 8 \\
\hline
\end{tabular}




\begin{tabular}{|c|c|c|c|c|}
\hline Autores & \multicolumn{4}{|c|}{ North FM. Syme SL. Feeney A. Shipley M. Marmot M. } \\
\hline Título & \multicolumn{4}{|c|}{$\begin{array}{l}\text { Psyhosocial work environment and sickness absence among british civil servants: The } \\
\text { Whitehall II study. }\end{array}$} \\
\hline Referencia & \multicolumn{4}{|c|}{ Am J Public Health. 1996; 86:332-340 } \\
\hline Diseño & \multicolumn{2}{|l|}{ Cohortes } & 9072 & \\
\hline Método & \multicolumn{4}{|c|}{ Adaptación del método de Karasek. } \\
\hline \multicolumn{5}{|l|}{ Resumen } \\
\hline \multicolumn{5}{|c|}{$\begin{array}{l}\text { Trabajadores no industriales (administración) del área de Londres participaron en el estudio. Tasa de } \\
\text { respuesta del } 73 \% \text {. Absentismo controlado en } 88 \% \text { de los trabajadores. Se clasificó el absentismo en }<7 \text { días } \\
\text { y }>7 \text { días. Demanda, control y soporte social en el ámbito laboral se asocian significativamente con elevadas } \\
\text { tasas de absentismo de (baja) y alta duración. }\end{array}$} \\
\hline OR - RR & \multicolumn{4}{|c|}{$\begin{array}{l}\text { (N=8307) Baja decisión: } 1,05(0,87-1,26) \text { Alta demanda: } 1,03(0,86-1,24) \\
+(\mathrm{N}=2886) \text { Baja decisión: } 0,91(0,75-1,10) \text { Alta demanda: } 1,34(1,12-1,60)\end{array}$} \\
\hline \multicolumn{5}{|c|}{ ESCALA DE NEWCASTLE-OTTAWA (Estudios Cohortes) } \\
\hline \multicolumn{5}{|c|}{\begin{tabular}{l|l} 
Selección & \\
\end{tabular}} \\
\hline \multirow{4}{*}{\multicolumn{2}{|c|}{$\begin{array}{l}\text { Representatividad de la cohorte } \\
\text { de expuestos }\end{array}$}} & \multicolumn{2}{|c|}{$\begin{array}{l}\text { Verdadera representatividad de los factores psicosociales en la } \\
\text { muestra* }\end{array}$} & \\
\hline & & \multicolumn{2}{|c|}{$\begin{array}{l}\text { Cierta representatividad de los factores psicosociales en la } \\
\text { muestra } *\end{array}$} & $\checkmark$ \\
\hline & & \multicolumn{2}{|c|}{ Grupos seleccionados de trabajadores (pacientes) } & \\
\hline & & \multicolumn{2}{|c|}{ No hay descripción } & \\
\hline \multirow{3}{*}{\multicolumn{2}{|c|}{$\begin{array}{l}\text { Selección de la cohorte de no } \\
\text { expuestos }\end{array}$}} & \multicolumn{2}{|c|}{ Elegida de la misma comunidad que la cohorte de expuestos* } & $\checkmark$ \\
\hline & & \multicolumn{2}{|c|}{ Elegida de un modo diferente } & \\
\hline & & \multicolumn{2}{|c|}{$\begin{array}{l}\text { No hay descripción de la selección de la cohorte de no } \\
\text { expuestos }\end{array}$} & \\
\hline \multirow{4}{*}{\multicolumn{2}{|c|}{ Conocimiento de la exposición }} & \multicolumn{2}{|c|}{ Fuente segura (p.e. registro quirúrgico)* } & \\
\hline & & \multicolumn{2}{|c|}{ Entrevista estructurada* } & \\
\hline & & \multicolumn{2}{|c|}{ Cuestionario autoadministrado } & $\checkmark$ \\
\hline & & \multicolumn{2}{|c|}{ No hay descripción } & \\
\hline \multirow{2}{*}{\multicolumn{2}{|c|}{$\begin{array}{l}\text { Demostración de que el evento } \\
\text { de interés no estaba presente al } \\
\text { inicio del estudio }\end{array}$}} & \multicolumn{2}{|l|}{$\mathrm{Si}^{*}$} & $\checkmark$ \\
\hline & & \multicolumn{2}{|l|}{ No } & \\
\hline \multicolumn{5}{|c|}{ Comparabilidad } \\
\hline & & Análisis de las cohortes & iodemográficos* & \\
\hline en la base do & seño o el análisis & $\begin{array}{l}\text { Estudio de controles por } \\
\text { laborales y educacionale }\end{array}$ & ional* (Factores & $\checkmark$ \\
\hline Resultado & & & & \\
\hline & & Ciego independiente* & & \\
\hline Conocimient & la exposición & Acoplamiento de registr & ción de la baja) & $\checkmark$ \\
\hline & & Auto informe & & \\
\hline & & Sin descripción & & \\
\hline $\begin{array}{l}\text { Fue el segui } \\
\text { suficienteme }\end{array}$ & $\begin{array}{l}\text { to } \\
\text { largo para que }\end{array}$ & $\begin{array}{l}\mathrm{Si} \text { (seleccionar un perioc } \\
\text { resultado esperado }>=6\end{array}$ & $\begin{array}{l}\text { to adecuado para el } \\
\text { os-dos meses) }\end{array}$ & $\checkmark$ \\
\hline sucediera el & & No $(<=6$ meses $)$ & & \\
\hline & & Seguimiento completo - & duos* & \\
\hline Idoneidad d & guimiento de las & $\begin{array}{l}\text { Pérdida de sujetos insuf } \\
\text { Pequeño número de pér }\end{array}$ & ducir un sesgo. & $\checkmark$ ? \\
\hline & & Tasa de pérdidas $>\%, s$ & e estas pérdidas & $\sqrt{ } ?$ \\
\hline & & Sin referencias & & \\
\hline Total & & & & $6 / 7$ \\
\hline
\end{tabular}




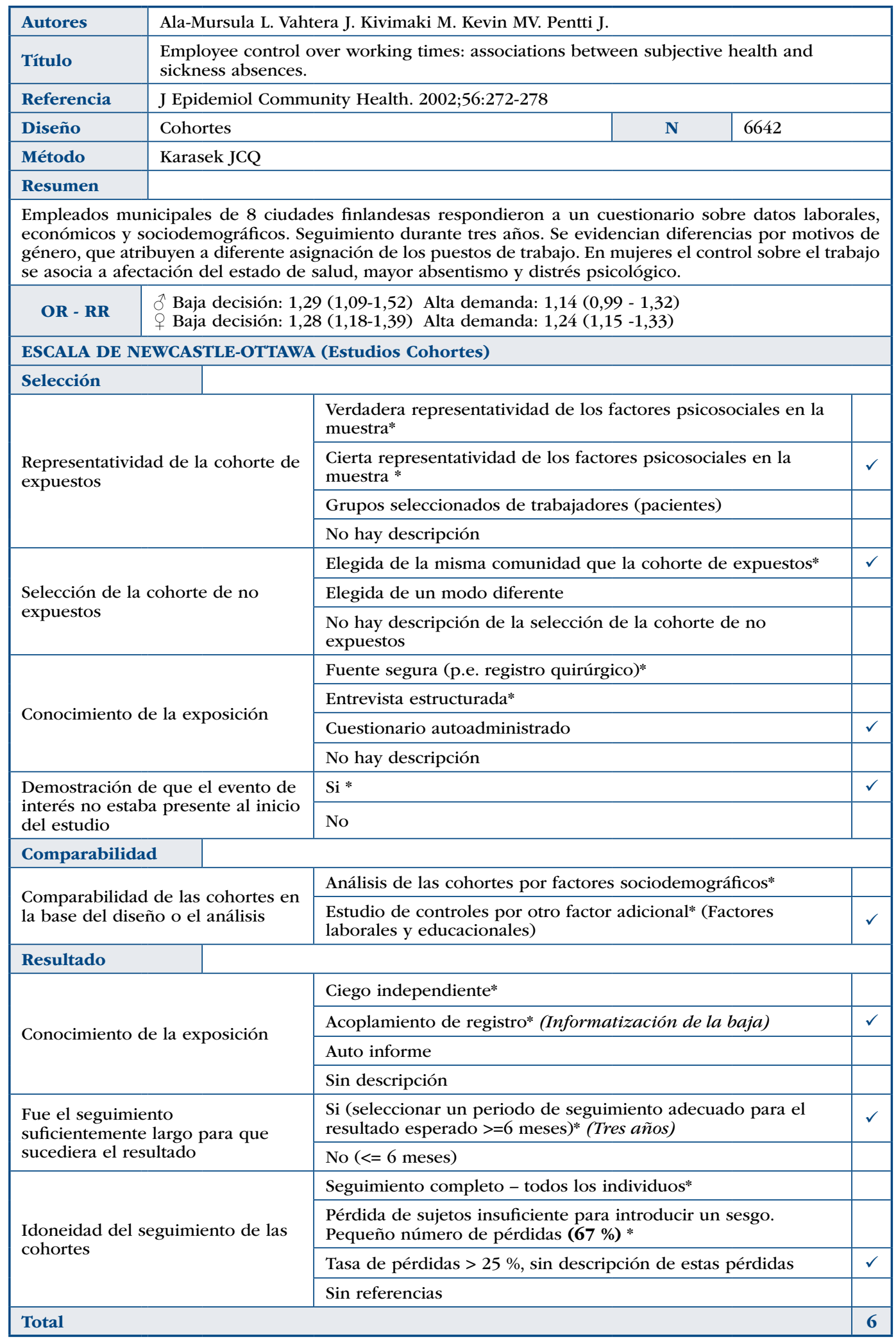




\begin{tabular}{|c|c|c|c|c|c|}
\hline Autores & \multicolumn{5}{|c|}{ Labriola M. Lund T. Burr H. } \\
\hline Título & \multicolumn{5}{|c|}{ Prospective study of physical and psychosocial risk factor for sickness absence. } \\
\hline Referencia & \multicolumn{5}{|c|}{ Occupational Medicine. 2006; 56:469-474. } \\
\hline Diseño & \multicolumn{2}{|l|}{ Cohortes } & I & 5574 & \\
\hline Método & \multicolumn{5}{|c|}{ Adaptació JCQ "ad hoc" } \\
\hline \multicolumn{6}{|l|}{ Resumen } \\
\hline \multicolumn{6}{|c|}{$\begin{array}{l}\text { Muestra aleatoria de trabajadores daneses, a los que se controlaron las bajas cortas/ largas ( }<6 \text { días/ }>6 \text { días). } \\
\text { El absentismo se asoció con la falta de control en el trabajo de manera significativa. }\end{array}$} \\
\hline OR - RR & \multicolumn{5}{|c|}{ 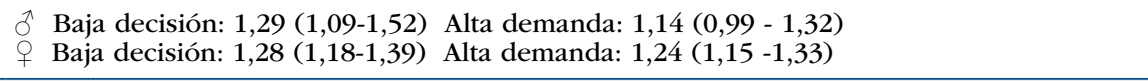 } \\
\hline \multicolumn{6}{|c|}{ ESCALA DE NEWCASTLE-OTTAWA (Estudios Cohortes) } \\
\hline \multicolumn{6}{|l|}{ Selección } \\
\hline \multirow{4}{*}{\multicolumn{2}{|c|}{$\begin{array}{l}\text { Representatividad de la cohorte de } \\
\text { expuestos }\end{array}$}} & \multicolumn{4}{|c|}{$\begin{array}{l}\text { Verdadera representatividad de los factores psicosociales en la } \\
\text { muestra* }\end{array}$} \\
\hline & & \multicolumn{3}{|c|}{$\begin{array}{l}\text { Cierta representatividad de los factores psicosociales en la } \\
\text { muestra * }\end{array}$} & $\checkmark$ \\
\hline & & \multicolumn{3}{|c|}{ Grupos seleccionados de trabajadores (pacientes) } & \\
\hline & & \multicolumn{3}{|c|}{ No hay descripción } & \\
\hline \multirow{3}{*}{\multicolumn{2}{|c|}{$\begin{array}{l}\text { Selección de la cohorte de no } \\
\text { expuestos }\end{array}$}} & \multicolumn{3}{|c|}{ Elegida de la misma comunidad que la cohorte de expuestos* } & $\checkmark$ \\
\hline & & \multicolumn{3}{|c|}{ Elegida de un modo diferente } & \\
\hline & & \multicolumn{3}{|c|}{$\begin{array}{l}\text { No hay descripción de la selección de la cohorte de no } \\
\text { expuestos }\end{array}$} & \\
\hline \multirow{4}{*}{\multicolumn{2}{|c|}{ Conocimiento de la exposición }} & \multicolumn{3}{|c|}{ Fuente segura (p.e. registro quirúrgico)* } & \\
\hline & & \multicolumn{3}{|c|}{ Entrevista estructurada* } & $\checkmark$ \\
\hline & & \multicolumn{3}{|c|}{ Cuestionario autoadministrado } & \\
\hline & & \multicolumn{3}{|c|}{ No hay descripción } & \\
\hline \multirow{2}{*}{\multicolumn{2}{|c|}{$\begin{array}{l}\text { Demostración de que el evento de } \\
\text { interés no estaba presente al inicio } \\
\text { del estudio }\end{array}$}} & \multicolumn{3}{|c|}{$\mathrm{Si} *$} & $\checkmark$ \\
\hline & & No & & & \\
\hline Comparabilic & & & & & \\
\hline & & Análisis de las & od & ficos* & \\
\hline la base del di & no o el análisis & $\begin{array}{l}\text { Estudio de co } \\
\text { laborales y ed }\end{array}$ & & tores & $\checkmark$ \\
\hline Resultado & & & & & \\
\hline & & Ciego indeper & & & \\
\hline Conocimiento & e la exnosición & Acoplamiento & ió & aja) & \\
\hline 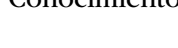 & ela exposicion & Auto informe & & & $\checkmark$ \\
\hline & & Sin descripció & & & \\
\hline $\begin{array}{l}\text { Fue el seguim } \\
\text { suficientemen }\end{array}$ & $\begin{array}{l}\text { nto } \\
\text { largo para que }\end{array}$ & $\begin{array}{l}\text { Si (selecciona } \\
\text { resultado espe }\end{array}$ & & o para el & $\checkmark$ \\
\hline sucediera el $r$ & & No $(<=6$ mes & & & \\
\hline & & Seguimiento c & du & & \\
\hline Idoneidad del & eguimiento de las & $\begin{array}{l}\text { Pérdida de suj } \\
\text { Pequeño núm }\end{array}$ & & esgo. & $\checkmark$ \\
\hline & & Tasa de pérdi & est & das & \\
\hline & & Sin referencia & & & \\
\hline Total & & & & & 7 \\
\hline
\end{tabular}




\begin{tabular}{|c|c|c|c|c|c|}
\hline Autores & \multicolumn{5}{|c|}{$\begin{array}{l}\text { Mastrangelo G, Mattioli S, Baldasseroni A, Bontadi D, Capodicasa E, Marzia V, Mazzi M, } \\
\text { Patané P, Torri P, Marangi G, Fadda E, Priolo G, Scoizzato L, Maier E, Campo G, Marchiori L. }\end{array}$} \\
\hline Título & \multicolumn{5}{|c|}{$\begin{array}{l}\text { Occupational injuries and sickness absence: association with job demand, decision latitude, } \\
\text { and life style in } 2174 \text { workers in the Veneto Region. }\end{array}$} \\
\hline Referencia & \multicolumn{5}{|c|}{ Med Lav. 2008;99 Suppl 1:31-41. } \\
\hline Diseño & \multicolumn{3}{|c|}{ Casos-controles } & 2174 & \\
\hline Resumen & \multicolumn{5}{|c|}{$\begin{array}{l}\text { Mediante el test de Karasek, se determina el riesgo psicosocial de } 2174 \text { trabajadores de } \\
30 \text { empresas de } 10 \text { a } 500 \text { trabajadores del Veneto italiano, pertenecientes a los sectores } \\
\text { industria y servicios. La duración mínima de la incapacidad temporal fue de } 10 \text { días. }\end{array}$} \\
\hline OR & \multicolumn{5}{|c|}{$\begin{array}{l}\text { Baja decisión: } 1,6(1,1-2,2) \\
\text { Elevada demanda: } 1,5(1,0-2,2)\end{array}$} \\
\hline \multicolumn{6}{|c|}{ ESCALA DE NEWCASTLE-OTTAWA (Estudios Caso-Control) } \\
\hline \multicolumn{6}{|c|}{\begin{tabular}{l|l} 
Selección & \\
\end{tabular}} \\
\hline \multirow{3}{*}{\multicolumn{2}{|c|}{$\begin{array}{l}\text { ¿Es adecuada la definición } \\
\text { de caso? }\end{array}$}} & \multicolumn{3}{|c|}{$\begin{array}{l}\mathrm{Si} \text {, con validación independiente丸t (Requiere validación independiente } \\
\text { (p.e. > 1persona/ registro/ tiempo/ proceso para extraer información o } \\
\text { referencia a la fuente primaria, registro médico/ hospitalario o estudio } \\
\text { radiológico.) }\end{array}$} & $\checkmark$ \\
\hline & & \multicolumn{3}{|c|}{$\begin{array}{l}\text { Si, por ejemplo mediante registros vinculados o basado en informes } \\
\text { propios (Registros vinculados, p.e. mediante códigos ICD a la base de } \\
\text { datos o informes propios sin referencia a registros primarios) }\end{array}$} & \\
\hline & & \multicolumn{3}{|c|}{ No hay descripción } & \\
\hline \multirow{2}{*}{\multicolumn{2}{|c|}{$\begin{array}{l}\text { Representatividad de los } \\
\text { casos }\end{array}$}} & \multicolumn{3}{|c|}{$\begin{array}{l}\text { Consecutiva o representatividad obvia de la serie de casos it (Todos } \\
\text { los casos con el evento de interés lo han sido en un periodo de tiempo, } \\
\text { en un espacio determinado, en un hospital, clínica, grupo hospitalario o } \\
\text { organización sanitaria, o bien ha sido una muestra de estos casos (p.e. } \\
\text { muestra aleatoria) }\end{array}$} & $\checkmark$ \\
\hline & & \multicolumn{3}{|c|}{$\begin{array}{l}\text { Sesgo de selección potencial o no indicado (No se satisfacen los } \\
\text { requerimientos del apartado anterior o no se indica) }\end{array}$} & \\
\hline \multirow{3}{*}{\multicolumn{2}{|c|}{ Selección de los controles }} & \multicolumn{3}{|c|}{ Controles comunitarios (empresas) is } & $\checkmark$ \\
\hline & & \multicolumn{3}{|c|}{ Controles hospitalarios } & \\
\hline & & \multicolumn{3}{|c|}{ No hay descripción } & \\
\hline \multirow{2}{*}{\multicolumn{2}{|c|}{ Definición de controles }} & \multicolumn{3}{|c|}{$\begin{array}{l}\text { No historia de enfermedad (punto final) (Si es el primer episodio de } \\
\text { enfermedad, debe explicitarse que no ha sucedido anteriormente) is }\end{array}$} & $\checkmark$ \\
\hline & & \multicolumn{3}{|c|}{ No descripción de la fuente } & \\
\hline \multicolumn{6}{|c|}{ Comparabilidad } \\
\hline \multirow{2}{*}{\multicolumn{3}{|c|}{$\begin{array}{l}\text { Comparabilidad de los casos y controles } \\
\text { en la base del diseño o el análisis }\end{array}$}} & \multicolumn{2}{|c|}{$\begin{array}{l}\text { Estudio de controles por edad y sexo (Seleccionar el } \\
\text { factor más importante) is }\end{array}$} & $\checkmark$ \\
\hline & & & \multicolumn{2}{|c|}{$\begin{array}{l}\text { Estudio de controles por otro factor adicional } \triangleq \text { (Este } \\
\text { criterio puede ser modificado indicando un control } \\
\text { específico por un segundo factor importante) }\end{array}$} & $\checkmark ?$ \\
\hline \multicolumn{6}{|l|}{ Exposición } \\
\hline \multirow{5}{*}{\multicolumn{3}{|c|}{ Conocimiento de la exposición }} & \multicolumn{2}{|c|}{ Fuente segura (p.e. registro quirúrgico) \& } & $\checkmark$ \\
\hline & & & \multicolumn{2}{|c|}{$\begin{array}{l}\text { Entrevista estructurada donde se ciega el estado de } \\
\text { casos y controles } \downarrow \text { ? }\end{array}$} & \\
\hline & & & Entrevista no cegada del estado & casos y controles & \\
\hline & & & Informe propio (auto registro) o r & stro médico & \\
\hline & & & Sin descripción & & \\
\hline Existe algún & odo para dis & cernir entre & $\mathrm{Si} i \mathrm{~s}$ & & $\checkmark$ \\
\hline & & & No & & \\
\hline Tasa de no $r$ & ndedores & & Tasa similar en ambos grupos is & & \\
\hline & & & Existe una descripción de los no & pondedores & $\checkmark$ \\
\hline & & & Tasa distinta sin designación & & \\
\hline Total & & & & & 7 \\
\hline
\end{tabular}

